\title{
Industrial Demands on Process and Device Simulation
}

\author{
A. v. Schwerin ${ }^{\mathrm{a}}$ and A. Spitzer ${ }^{b}$

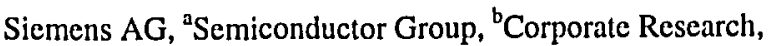 \\ Otto-Hahn-Ring 6, 81739 Munich, Germany \\ (invited paper)
}

\begin{abstract}
In this paper, we will give our view of the role of TCAD in the industrial technology development process, as well as of the division of labor between industrial TCAD, vendors of commercial TCAD software, and academia. Furthermore a list of model shortcomings is presented that - we fecl still - prevent broad productive use of TCAD in industrial technology development. In the end we will define our primary demands for future development work on process and device simulation by vendors and institutes and our vision of the role of TCAD frameworks.
\end{abstract}

\section{Introduction}

Technology CAD was always meant to be used by semiconductor industry for device design and manufacturing process development. Although pretty significant progress of models and tools has been made over the years, the actual application of process and device simulation in technology development still does not come up to ambitious expectations. We will discuss the reason for this somewhat disappointing situation as well as our demands and visions for the future of TCAD.

\section{The mission of industrial TCAD, the role of vendors and academia}

The mission of an industrial TCAD department is not just "to do simulation" or "to provide TCAD models" but to increase the productivity of the technology development process in microelectronics. Thus, a significant impact of TCAD application should be a reduction of development time, number of learning cycles, and development costs. Otherwise, there would be no investment in TCAD departments and software. To come 
up to these expectations two main tasks have to be accomplished. The first is to make TCAD reliable, i.e. looking after the proper models. The second is to promote and to support the application of TCAD programs as tools for the process development engineer.

About 10 years ago industry was mostly using (if at all) proprietary TCAD tools and/or programs directly provided by institutes from universities (e.g. MINIMOS [1], SUPREM [2], and PISCES [3]). The R\&D departments were mostly responsible for further development of models and tools. Since then a highly specialized software industry has developed that offers today programs (most of them still based on the university codes). Some of these programs have become a quasi-industry standard (e.g. TSUPREM-4 [4]). The reasons for paying a significant amount of money for this commercial software rather than using in-house or university tools for free are similar to those in other ficlds where software is used in industry. Especially in the TCAD area the demands of the semiconductor industry induce that generally accepted physical models delivered by universities are implemented into the tools along the line of the technology development. Based on this distribution of tasks, neither software support nor the implementation of other scientists' models are adequate tasks of universities or institutes. We see the main role of academia in the development of predictive physical models. In this context, the university codes [5-8] have still their raison d'être for test implementation and verification of new physical models. Therefore, the right way to make a new simulation model productive for technology development is a) to convince the community by publication, b) to promote the implementation of the new model in one of the vendor tools. Without step $b$, a new model will be of little use for industrial application. This might gradually change in the near future when flexible model interfaces (which are announced today) are available for the commercial programs. In Fig. 1 we try to illustrate this idea of a division of labor between academia, software vendors, and industrial TCAD.

\section{Model needs}

As mentioned above, we believe that the main challenge in TCAD development is still to provide adequate physical models for predictive simulation. Typical simulation accuracy requested in an industrial environment is e.g. to predict MOSFET drain currents (based on "default parameters") for a new process with relative deviation of less than 5\%. Today this is only possible after careful calibration to an existing technology.

In the following sections we will list some model shortcomings in the area of device and process models that prevent the TCAD user from being more predictive. This list covers a number of classical problems which in our view are still not solved satisfactorily at least in the frame of commercial simulation tools. We also have some new topics on our list which are hardly tackled at all in available programs. 


\section{Division of Labor for TCAD - Development}

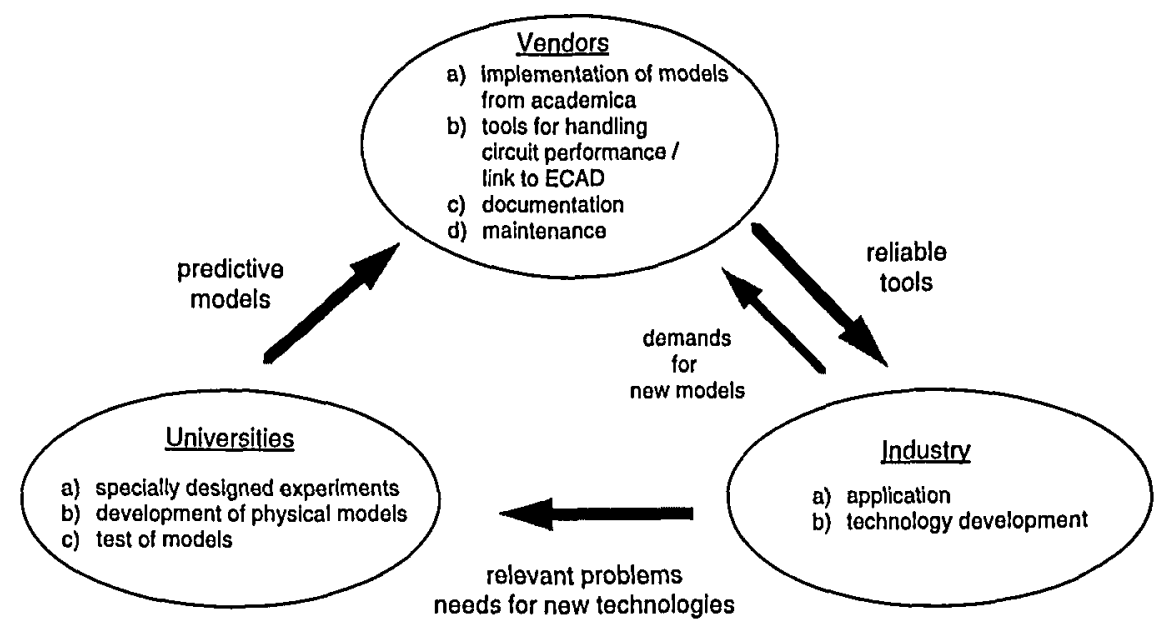

Fig. 1: Illustration of our view of the division of labor in TCAD development and application

\section{3..1 Device simulation problems}

The first two problems have a long tradition in device simulation. A lot of (sometimes very successful) modeling has been done in this field. However the solutions to be found in commercial software are pretty disappointing.

- Mobility in MOSFETs with thin gate oxides / high channel doping [9] including quantum mechanical corrections [10]

- Hot-carrier effects like substrate current, device degradation, and reliability. Actually a lot of modeling work has been done in this field. Nevertheless the models to be found in commercial simulation tools are still not at all sufficiently accurate even for mere substrate current calculation.

- Junction leakage current due to mechanical stress [11], dislocations or point defects

- Models for nonvolatile devices with ferroelectric [12] or ferromagnetic materials [13].

Turning from the single device to the circuit, hot topics are

- circuit reliability [14]

- parasitics extraction for large circuits with realistic non-rectangular interconnect geometry [15]

- worst case / circuit yield simulation [16].

For the single devices we mentioned modeling problems where we expect solutions from academia. On the other hand the circuit problems could be solved by the software houses. The underlying physics and methodology is rather well known. The problem is to handle quite complex systems of many interacting elements. The first commercial tools are 
available but have not yet been implemented into the standard tool flow of circuit design. Thus, one important task is to establish the link to the ECAD world.

\section{$3 . .2$ Process simulation problems}

As goes for the device modeling problems there are some promising solutions to be found in literature for some of the following topics, especially the classical ones, but no consistent modeling is available in state-of-the-art commercial software.

- Correct modeling of dopant profiles especially at material boundaries [17], at high concentration [18] or after ion implantation [19]

- Doping activation and deactivation phenomena

- Amorphization and recrystallization [20]

- Mechanical stress and dislocation formation [21, 22]

- Processing and geometry dependence of the hysteretic properties of ferroelectric and ferromagnetic materials [23]

- Modeling of so called „isolated-nested" effects, i.e. „loading effects“ during plasma deposition and etch [24]

- Chemical mechanical polishing [25].

The last two topics bear a common conceptual difficulty. To solve these problems the medium range vicinity of the actually simulated (microscopic) part of the wafer has to be taken into account. For a solution, concepts of the conventional process simulation (typical scale: $1-10 \mu \mathrm{m}$ ) and strategies and know how of the equipment simulation

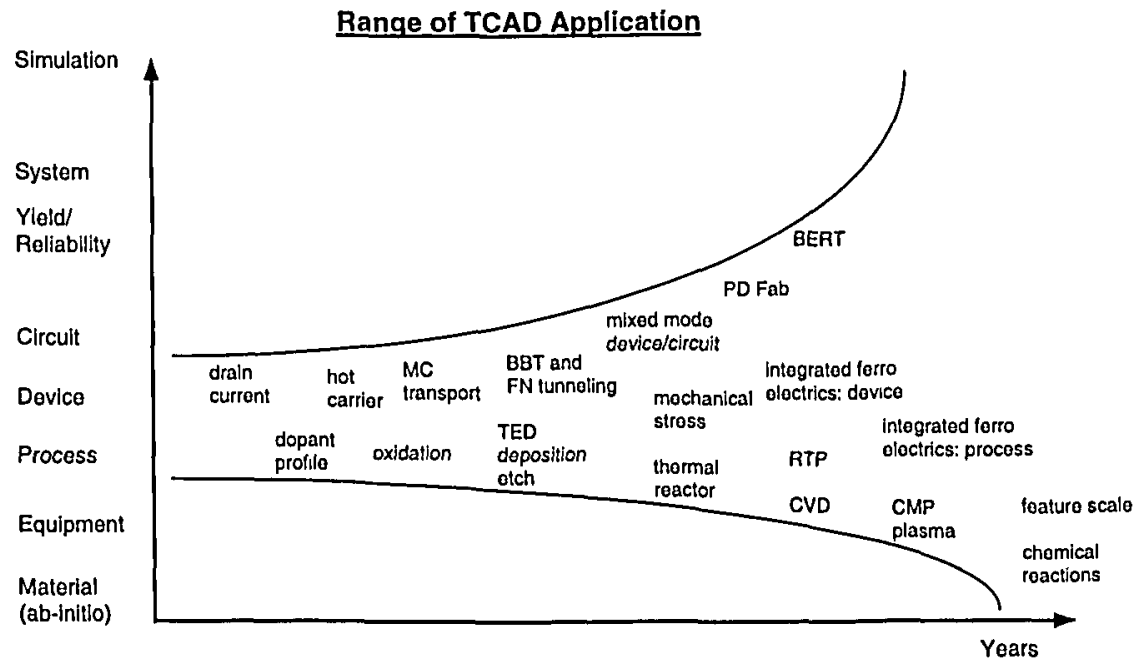

Fig. 2: Increasing range of TCAD application through the years. In the past, TCAD was mainly focused to a narrow range of process and device problems. With increasing CPU performance and with increasing modeling experience the scope broadened and should make TCAD really successful. 
(typical scale: $10-100 \mathrm{~cm}$ ) must merge together in order to extend process simulation into the new areas.

Problems to be solved mainly by vendors in the field of process simulation are

- 3D simulation

- adaptive gridding that really works.

Fig. 2 illustrates the increasing range of TCAD application through history projected into the future.

\section{Hierarchy of simulation tools}

We are aware of the fact that a number of the modeling topics listed above have been or can be solved today with satisfactory accuracy, but only with excessive cost in terms of CPU time (e.g. Monte Carlo (MC) or molecular dynamics (MD) codes). Thus, these solutions are not useful for technology development or process latitude analysis. Nevertheless, these programs play an important role in the value chain (academia - SW vendor - industrial simulation departments - process engineer). They provide a means to virtually ,measure“ quantities which are not measurable with sufficient accuracy in physical reality (carrier distributions, $2 D$ and 3D impurity distributions, activation energies, etc.). Thus, the results of these more accurate but very slow programs can be used to tune heuristic CPU efficient models. These heuristic models are then suitable for

\section{Hierarchy of Simulation Tools}

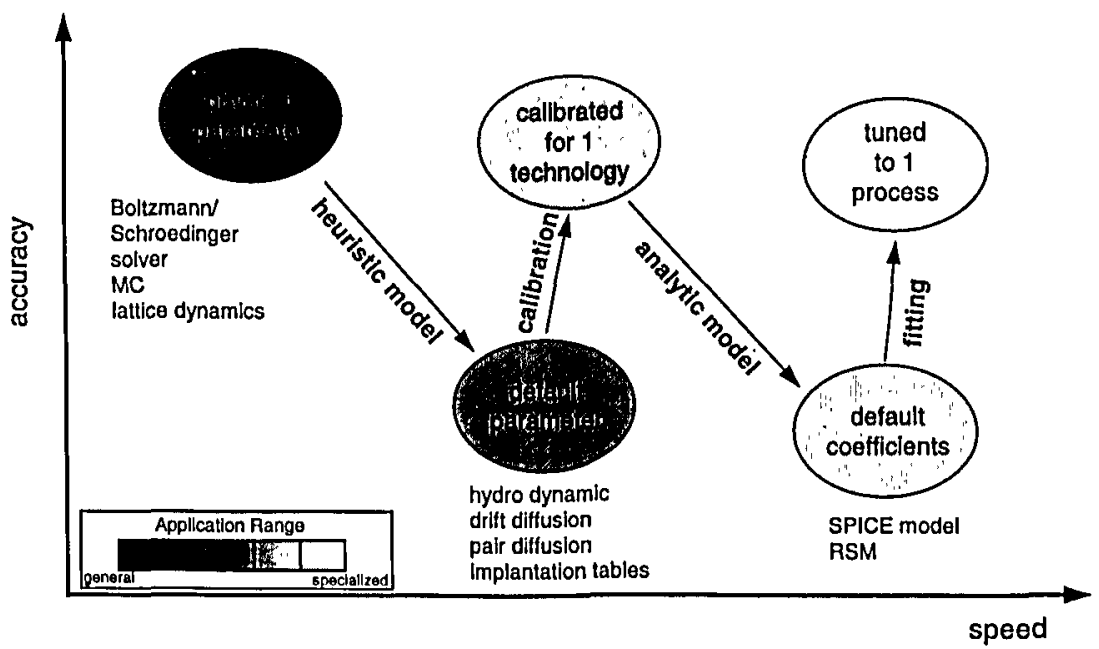

Fig. 3: Trade-off of speed and accuracy in the hierarchy of simulation tools 
implementation into commercial software. This makes it possible after all to use the results of very complex and time consuming programs for technology development. The same is true for simple response surface models (RSM) which make the results of still too time consuming 2D process and device simulations available on the $\mathrm{PC}$ of the device engineer for fine tuning of the process for optimum performance etc.. Fig. 3 illustrates the trade-off of speed and accuracy in the hierarchy of simulation tools.

\section{Vision of industrial use of TCAD}

TCAD frameworks can possibly play an important role in making TCAD available for the non-expert. The typical user of such a framework would be a device engineer or even a circuit designer. The framework should enable him to use TCAD as naturally as he uses a range table, a pocket calculator or an Excel sheet today.

Thus, the task of a TCAD framework should be first to ease the use of TCAD tools including possibilities for design of experiments and optimizations (as today already partly realized in available systems). Second and even more important, it should reduce the risk of false usage of these tools to a minimum and make every result completely retrievable! This framework together with the implemented tools represents an expert system for silicon processing.

However, we still see the open model questions as the main blocking point. Prerequisite for a productivity enhancement by the use of TCAD frameworks is that models are sufficiently accurate and a number of unique rules can be formulated on how to calibrate and use these models correctly. Only then process or device simulation modules can be defined which are safe to use and which can be exchanged without the risk of wrong results.

\section{Conclusion}

Although a lot of progress has been done in the last decade, we are still far from the "brave new world" of plug and play TCAD tools. Still a number of old modeling problems are open and new modeling tasks come up with every new key processing step or material. We suggest a clear division of tasks between academia (model development, providing results from virtual or sophisticated real experiments for heuristic model calibration) and software vendors (develop and support complex tools). Industrial TCAD groups should provide the requirements for current and future technologies as well as a conceptional structure of a TCAD framework. We will have reached our goal when the 
technology development engineer will use the tools as naturally and successfully as a circuit designer uses SPICE simulation and parasitics extraction today.

\section{References}

[1] S. Selberherr, A. Schuetz, and H. Poetzl, "MINIMOS - A two dimensional MOS transistor analyzer," IEEE Trans. Electron Devices, vol. ED-27, pp. 1540-1550, 1980.

[2] M. E. Law, "Two dimensional numerical simulation of dopant diffusion in silicon," Ph.D. dissertation, Stanford University, Stanford, CA, 1988.

[3] M. R. Pinto, C. S. Rafferty, H. R. Yeager, and R. W. Dutton, "PISCES-IIB - Poisson and continuity equation solver," Stanford Electronics Lab. Tech. Rep., Stanford Univ., Feb., 1986.

[4] "TSUPREM-4, Two-dimensional process simulation program," Version 6.5, User's Manual, Technology Modeling Associates, May, 1997.

[5] C.-C. Lin and M. Law, "2-D adaptive simulation of dopant implantation and diffusion," Proc. of SISPAD, vol. 6, pp. 282-285, Erlangen, 1995.

[6] D. Yergeau, E. Kan, M. Gander, and R. Dutton, "ALAMODE: A layered model development environment," Proc. of SISPAD, vol. 6, pp. 66-69, Erlangen, 1995.

[7] J. Litsios, B. Schmithusen, and W. Fichtner, "Large scale thermal mixed mode device and circuit simulation," Proc. of SISPAD, vol. 6, pp. 368-371, Erlangen, 1995.

[8] T. Simlinger, H. Kosina, M. Rottinger, and S. Selberherr, "MINIMOS-NT: A generic simulator for complex semiconductor devices," Proc. ESSDERC, pp. 83-86, 1995.

[9] S. Villa, A. Lacaita, L. Perron, and R. Bez, "A physically-based model of the effective mobility in heavily-doped n-MOSFETs," IEEE Trans. Electron Devices, vol. ED-45, pp.110-115, 1998.

[10] M. van Dort, P. Woerlee, A. Walker, C. Juffermans, and H. Lifka, "Influence of high substrate doping levels on the threshold voltage and the mobility of deep-sub $\mu \mathrm{m}$ MOSFETs," IEEE Trans. Electron Devices, vol. ED-39, pp.932-938, 1992.

[11] P. Smcys, P. Griffin, and K. Saraswat, "Influence of post-oxidation cooling rate on residual stress and pn-junction leakage current in LOCOS isolated structures," IEEE Trans. Electron Devices, vol. ED-43, pp. 1989-1993, 1996.

[12] R. Jones, Jr., P. Maniar, R. Moazzami, P. Zurcher, J. Witowski, Y. Lii, P. Chu, and S. Gillespie, "Ferroclectric non-volatile memories for low-voltage, low-power applications," Proc. on Thin Solid Films, vol. 270, pp. 584-585, San Diego,CA, 1995.

[13] D. Tang, P. Wang, V. Speriosu, S. Le, R. Fontana, and S. Rishton, "An IC process compatible nonvolatile magnetic RAM," IEDM Tech. Dig., pp. 997-1000, Washington, DC, 1995. 
[14] K. Quader, E. Minami, Wei-Jen Ko, P. Ko, and Chenming Hu, "Hot-carrier-reliability design guidelines for CMOS logic circuits," IEEE J. Sol.-State Circuits, vol.29, pp.253-262, 1994.

[15] Ching-Chao Huang, Kyung Suk Oh, Shun-Lien Wang, and S. Panchapakesan, "Improving the accuracy of on-chip parasitic extraction," Proc. on Electrical Performance of Electronic Packaging, pp. 42-45, San Jose, CA, 1997.

[16] J. Power, B. Donnellan, A. Mathewson, and W. Lane, "Relating statistical MOSFET model parameter variabilities to IC manufacturing process fluctuations enabling realistic worst case design," IEEE Trans.Semicon. Manufact. vol.7, p.306-318, 1994.

[17] F. Lau, L. Mader, C. Mazure, C. Werner, and M. Orlowski, "A model for phosphorus segregation at the silicon-silicon dioxide interface," Appl. Phys. A vol. A49, pp.671$675,1989$.

[18] S. Dunham, "A quantitative model for the coupled diffusion of phosphorus and point defects in silicon," J. Electrochem. Soc., vol. 139, pp. 2628-2636, 1992.

[19] P. Griffin, R. Lever, R. Huang, H. Kennel, P. Packan, and J. Plummer, "Species, dose and energy dependence of implant induced transient enhanced diffusion," IEDM Tech. Dig., pp. 295-298, Washington, DC, 1993

[20] H. Chao, P. Griffin, J. Plummer, "The influence of amorphizing implants on boron diffusion in silicon," Proc. of the MRS Symp., pp. 347-352, San Francisco, CA, 1997.

[21] H. Ho,E. Hammerl, R. Stengl, and J. Benedict, "Dislocation formation in trench based dynamic random access memory (DRAM) chips," Proc. of the MRS Symp., pp. 459-466, Boston, MA, 1995.

[22] M. Dellith, G. Booker, B. Kolbesen, W. Bergholz, and F. Gelsdorf, "A dislocation formation model of trench-induced dislocations in dynamic random access memories," J. Electrochem. Soc., vol. 143, pp. 210-215, 1996.

[23] S. Kobayashi, N. Tanabe, Y. Maejima, Y. Hayashi, and T. Kunio, "Scaling possibility of PZT capacitors for high density and low-voltage NVFRAM application," Integ. Ferroelectrics, vol. 17, pp. 81-88, Santa Fe, NM, 1997.

[24] J. Lee, N. Layadi, K. Guinn, H. Maynard, F. Klemens, D. Ibbotson, I. Tepermeister, P. Egan, and R. Richardson, "Comparison of advanced plasma sources for etching applications. V. Polysilicon etching rate, uniformity, profile control, and propertics in a helical resonator plasma source," J. Vac. Sci. \& Tech.B, vol. 14, pp. 2510-2518, 1996.

[25] Kim Yoo-Hyon, Kim Tai-Kyung, Lee Hoong-Joo, Kong Jeong-Tack, and Lee SangHoon, "CMP profile simulation using an elastic model based on nonlinear contact analysis," Proc. of SISPAD, pp. 69-72, Cambridge, MA, 1997. 